\title{
COORDINATION OF ARM AND WRIST MOTION DURING A REACHING TASK ${ }^{1}$
}

\author{
F. LACQUANITI AND J. F. SOECHTING ${ }^{2}$
}

Laboratory of Neurophysiology, University of Minnesota Medical School, Minneapolis, Minnesota 55455

Received September 8, 1981; Revised November 9, 1981; Accepted November 25, 1981

\begin{abstract}
An analysis of arm movements involving forward projection of the hand in order to reach for and grasp a target at different orientations is presented. The reaching movements required shoulder flexion, elbow extension, and wrist pronation or supination. The relation between elbow and shoulder instantaneous angular position proved to be consistent from trial to trial of each task, independent of movement speed. Further, this relation was not influenced by the presence or absence of a concomitant wrist rotation. During the deceleratory phase of the movement, the slope of elbow angular velocity to shoulder angular velocity was constant and independent of target orientation. Wrist motion was instead highly variable in timing, course, and duration. Supinatory movements tended to be fractionated. On average, the duration of wrist movements was shorter than that of shoulder and elbow motions. The pattern of biceps EMG activity during supinatory and pronatory movements was different. Since motion at the shoulder and elbow was virtually identical in the two cases, net flexor torque at the elbow was also little different. It is concluded that other elbow flexors and extensors also exhibit a lask-dependent patterning of activity so as to produce the same net torque.

The results are discussed in the context of the internal constraints present during the movements that we examined. These constraints are the inertial coupling between shoulder and elbow motion and those which derive from the bifunctional nature of many of the muscles participating in the movement.
\end{abstract}

Motor coordination requires the mastering of a large number of degrees of freedom of the musculoskeletal apparatus so as to reduce the indeterminacy of the system without compromising the ability to adapt to the different demands of specific motor tasks (Bernstein, 1967).

In order to begin to understand the problem of motor coordination, one may look at the manner in which some simple motor tasks are executed with the hope of discovering general laws which govern the spatiotemporal evolution of such movements. Thus, in a previous paper (Soechting and Lacquaniti, 1981), we showed that kinematic invariances do characterize pointing movements of the arm. Namely, the relation between elbow and shoulder instantaneous angular position is consistent in different trials and is independent of the overall speed of the movement to a specified target; secondly, the slope of elbow angular velocity versus shoulder angular velocity

\footnotetext{
'This work was supported by United States Public Health Service Grant NS-15018 and National Science Foundation Grant BNS-8117625.

${ }^{2}$ To whom correspondence should be addressed at Laboratory of Neurophysiology, 5-257 Millard Hall, University of Minnesota Medical School, 435 Delaware Street Southeast, Minneapolis, MN 55455.
}

is constant and independent of the target location during the terminal phase of the movement.

The problem of the extent to which these invariances can be generalized to other tasks, however, remains open. Two specific questions can be raised in this context. The first is whether the relation between elbow and shoulder motions observed in movements with only two degrees of freedom remains the same when additional degrees of freedom are introduced in the system, for example, when motions at the wrist and hand also come into play in the execution of the task.

The second question concerns the possibility that there exist invariant relationships involving other degrees of freedom of the arm. For instance, Kots and Syrovegin (1966) studied motor tasks where the subjects were instructed to carry out movements involving simultaneous rotation at the elbow and at the wrist in all possible combinations of flexion-extension. They claimed to have found periods of movements during which the two joints were displaced with a constant ratio of their angular velocities; furthermore, the set of ratios for each subject was limited. Bishop and Harrison (1977), however, were unable to reproduce these findings.

In order to address these two questions, we have 


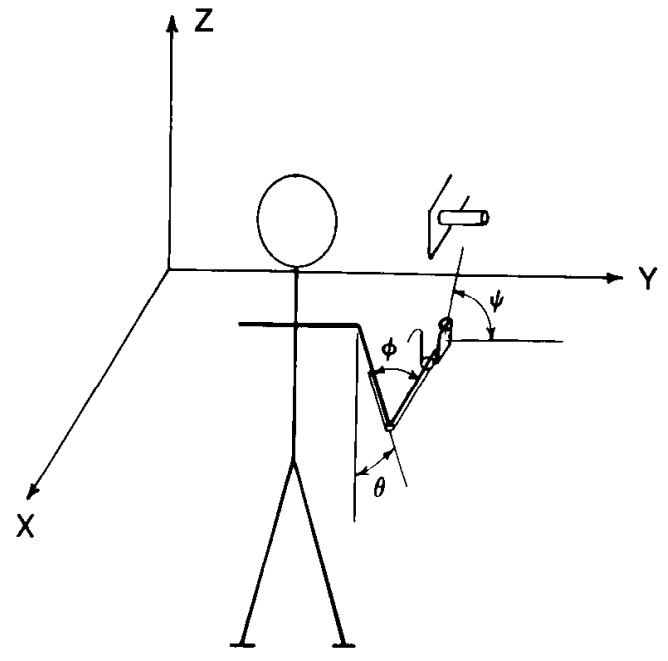

Figure 1. Schematic of the experimental set-up. Arm movements were executed in the parasagittal plane and directed to grasp a cylinder at different orientations in the frontal plane. Elbow extension (angle $\phi$ ) and wrist pronation-supination (angle $\psi$ ) were measured electrogoniometrically. Forward flexion at the shoulder (angle $\theta$ ) was calculated indirectly by measuring instantaneous wrist position in three-dimensional coordinates $(X, Y$, and $Z$ ) by means of an ultrasound source.

studied a task which required reaching for and grasping a handle having different orientations. Under the experimental conditions imposed, these movements involve the coordination of the angular motions at the shoulder (forward flexion), elbow (extension), and wrist (pronation or supination).

\section{Materials and Methods}

Motor task. This report summarizes the results of eight experiments involving four subjects. They were instructed to reach for and grasp a cylinder (Fig. 1) which could be positioned at different angular orientations in the frontal plane. Each subject stood erect at a distance which permitted him to reach the cylinder by means of a forward projection of his arm in the parasagittal plane. All movements were initiated with the upper arm approximately vertical and the forearm approximately horizontal and either supinated or half-pronated according to the specific task. Therefore, while the direction and the total angular excursion of the shoulder and elbow movements were approximately constant throughout the experiment, the direction and amount of forearm rotation varied as a function of the initial position of the hand and of the orientation of the cylinder. Each experimental session consisted of at least 50 movements, some requiring pronation or supination of the wrist by as much as $90^{\circ}$ as well as others requiring no change in orientation of the wrist. The subjects were instructed to initiate movement upon hearing a brief tone; they were asked to vary its speed from trial to trial. No instructions were given regarding the manner in which the task was to be accomplished.

Recording system and data analysis. The changes in elbow angle and in wrist angle were measured electrogoniometrically. The principles and the design of the triaxial goniometer which was used have been detailed by Chao (1980) and by Chao et al. (1980). The goniometer consists of a universal joint at the elbow and a circular brace at the wrist. It was strapped to the upper arm and the inner ring of the circular brace was clamped to the forearm proximal to the wrist. Since goniometric methods are less well suited to measure movement about the shoulder joint (Chao, 1978, 1980; Dvir and Berme, 1978), we resorted to indirect methods to estimate the angle of forward flexion $(\theta)$ by measuring the position of the wrist in three-dimensional space. This was accomplished by means of an ultrasound-emitting device which was attached to the outer ring of the brace and aligned with the midpoint of the axis through the distal ends of the radius and ulna. The position of the ultrasound source was unaffected by forearm rotation, and its distance to three orthogonal linear microphones ( $X, Y$, and $Z$ in Fig. 1) was measured with a resolution of $0.1 \mathrm{~mm}$ at a sampling rate of $100 \mathrm{~Hz}$. The shoulder angle $\theta$ then was derived trigonometrically (see Soechting and Lacquaniti, 1981, for details). This procedure assumed that the movement was restricted to the sagittal plane and involved no abduction or internal axial rotation at the glenohumeral joint. Neglecting the motion of these two degrees of freedom, which did occur under some experimental conditions, contributed to the error in estimating the amount of forward flexion at the shoulder as did the assumption that motion at that joint consisted of a pure rotation. The error in the estimate of $\theta$ introduced by these factors never exceeded $5^{\circ}$ or $10 \%$ of the excursion in $\theta$.

Following double-sided exponential smoothing, angular and linear displacements were differentiated numerically to obtain velocity and acceleration. In order to compare the profiles of angular velocities corresponding to movements of different speed, $\dot{\phi}(t), \dot{\theta}(t)$, and $\dot{\psi}(t)$ were normalized with respect to time in the following manner. Movement duration at a given joint was defined as the time during which its angular velocity exceeds $10 \%$ of peak angular velocity. Angular velocity profiles at the shoulder, elbow, and wrist were normalized in time relative to the duration of forward flexion at the shoulder.

We did not attempt to provide a complete description of the muscular activities involved (cf., Basmajian, 1978) and, instead, selected a few representative muscles (deltoid, biceps, and pronator teres) whose EMG activity was recorded by means of surface electrodes.

\section{Results}

Figure 2 shows the results of two single trials from one subject: in part $A$, the task required wrist pronation in order to grasp the handle positioned vertically, while in part $B$, wrist supination with the handle horizontal was required. Prior to the onset of the movement, each subject stood with his arm at his side (shoulder angle $\theta$ approximately equal to $0^{\circ}$ ), his forearm horizontal (elbow angle $\phi$ about $90^{\circ}$ ), and either supinated (wrist angle $\psi$ about $0^{\circ}$ ) or half-pronated ( $\psi$ about $90^{\circ}$ ), and his hand semiflexed. The movements illustrated were of medium speed with a duration of about 600 msec. The first two traces depict the linear motion of the source located at the wrist in the horizontal $(X)$ and in the vertical direction $(Z)$. ( $Y$ remained constant throughout, because the movement occurred in a plane perpendicular to its axis.) 

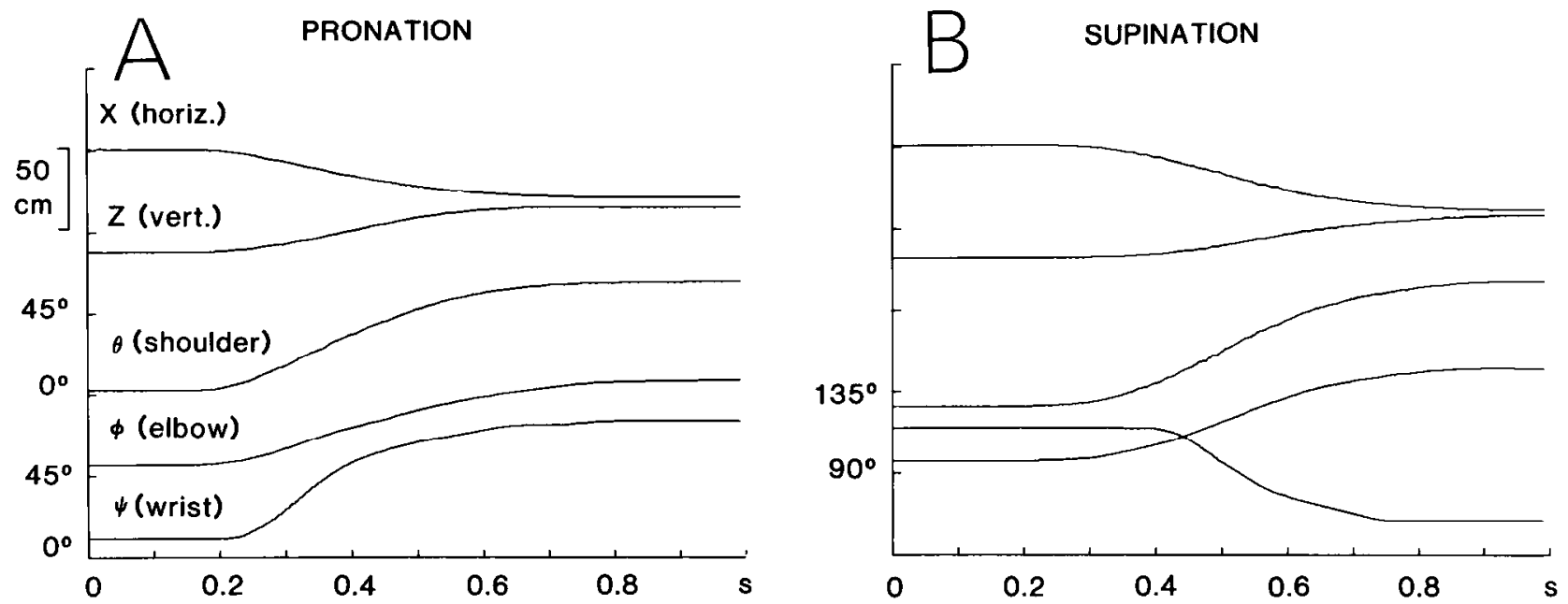

Figure 2. Representative examples of reaching movements involving wrist pronation $(A)$ and supination $(B)$. The traces from top to bottom correspond to horizontal and vertical Cartesian coordinates of the ultrasound source located at the wrist, shoulder angle $\theta$, elbow angle $\phi$, and wrist angle $\psi$. The scale for $\phi$ is given in $B$.

The next three traces describe the forward flexion at the shoulder $(\theta)$, elbow extension $(\phi)$, and forearm rotation $(\psi)$, respectively.

One would expect the final value of the angle $\psi$ to be about $90^{\circ}$ in Figure $2 A$ and about $0^{\circ}$ in Figure $2 B$, given the relative orientations of the handle; instead, $\psi$ is $71^{\circ}$ and $19^{\circ}$, respectively. The reason for this discrepancy (which was found consistently) is the following. The reaching movements involved more degrees of freedom than those described above. In the course of movements involving pronation and directed to the vertical target, a small amount of abduction and internal axial rotation occurred at the glenohumeral joint, resulting in an internal rotation of the forearm and contributing to the orientation of the hand. Moreover, in both the movements involving pronation and in those involving supination, flexion of the hand and some degree of flexion-extension and abduction-adduction at the wrist at the end of the reaching movement allowed an orientation of the palm of the hand adequate to grasp the handle without any need for a perfect alignment of the wrist with the handle. However, in each task, the final values of $\theta, \phi$, and $\psi$ were reproducible from trial to trial for a given subject.

We shall describe the kinematic characteristics of these movements in more detail in the following section and shall focus on two questions: first, is the evolution of the motion at the more proximal joints affected by forearm rotation and target orientation? Note that some of the muscles involved in producing these movements are bifunctional; for example, the biceps muscle acts as an agonist during supination and as an antagonist to pronation in addition to its action as elbow flexor. How are these potentially conflicting actions resolved? The second question that we shall address is whether coupling between pronation-supination and motion at the more proximal joints can be demonstrated.

Relations between elbow and shoulder motions. We have shown previously (Soechting and Lacquaniti, 1981) that shoulder and elbow motions are tightly coupled for pointing movements involving these two degrees of freedom and not requiring a specific orientation of the wrist.
For such movements, the trajectory of the arm is invariant of movement speed. Furthermore, the movement is so organized that the trajectories described in the velocity space $\dot{\theta}, \dot{\phi}$ converge on a straight line in the deceleratory phase of the movement. The slope of this line is close to unity and is invariant of the vertical location of the target.

Both of these invariant characteristics of shoulder and elbow motion persist under the present experimental conditions. Specifically, they are affected neither by the presence of concomitant rotation of the forearm nor by the spatial orientation of the handle to be grasped (and therefore the final position of the hand). In order to separate these effects, we asked our subjects to perform reaching movements in the presence and absence of forearm rotation and also movements directed to differently oriented targets.

Figure 3 shows the trajectories produced by one subject under three different experimental conditions. The initial orientation of the hand, the direction of forearm rotation during the movement, and the target orientation are shown schematically at the left of each row. The trajectories of all of the trials performed by this subject under each of the three experimental conditions have been superimposed in Figure 3. They are represented in the Cartesian coordinate system ( $X, Z$-first column) and in the intrinsic coordinate system relating shoulder and elbow angles $(\theta, \phi-$ second column $)$ and in velocity space $(\dot{\theta}, \dot{\phi}$-third column). The latter also are shown after normalization with respect to maximum angular velocity at the shoulder. This parameter ranged from $80^{\circ} / \mathrm{sec}$ to $200^{\circ} / \mathrm{sec}$ in this experiment. Note that the relation between motion at the shoulder and at the elbow is very consistent from trial to trial and invariant of movement speed under each of the experimental conditions.

Furthermore, the presence of forearm rotation during the movements has no substantial effect on the trajectories at the shoulder and at the elbow (compare Fig. 3, $A$ to $D$ with $E$ to $H$ ). In both cases, the trajectories in the intrinsic velocity space representation (Fig. 3, $C$ and $G$ ) converge to a straight line in the deceleratory phase 

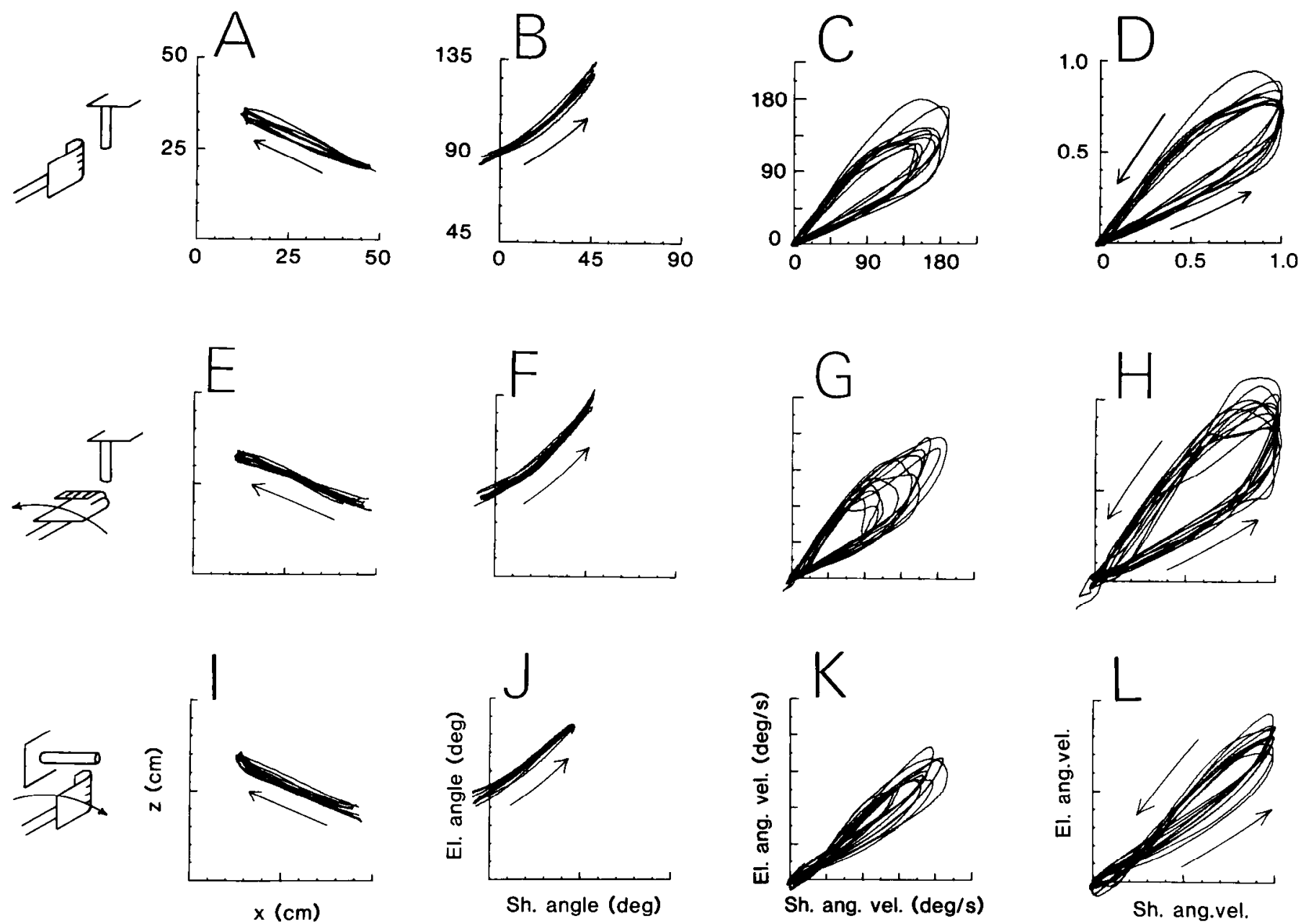

Figure 3. Movement trajectories in extrinsic and intrinsic coordinate systems. The schematics depict the initial position of the hand and the orientation of the cylinder to be grasped. The first row ( $A$ to $D$ ) presents the results obtained with the handle vertically oriented and the wrist semipronated, thus requiring no changes in wrist orientation; the target was oriented vertically with the hand initially supinated in $E$ through $H$; it was horizontal and the hand was initially half-pronated in $I$ through $L$. Individual trials performed by one subject have been superimposed. The first column ( $A, E$, and $I)$ shows the trajectory of the source located at the wrist in Cartesian coordinates $(X, Z)$, with the movement progressing in the direction of the arrow. The second column $(B, F$, and $J)$ shows, for the same trials, the relations between elbow and shoulder angles, and the third column $(C, G$, and $K$ ) depicts the relations between angular velocities at the two joints. These have been normalized with respect to maximum angular velocity at the shoulder in the fourth column $(D, H$, and $L)$. Notice the small variability among single trials in all representations for each task.

TABLE I

Slope and intercept of velocity space trajectories

\begin{tabular}{lcc} 
& \multicolumn{1}{c}{ Slope $^{a}$} & Intercept $^{a}$ \\
\hline Vertical target & & \\
$\quad$ Without wrist rotation & $1.13 \pm 0.13$ & 0 \\
With wrist pronation & $1.11 \pm 0.16$ & 0 \\
& & \\
Horizontal target & $1.08 \pm 0.13$ & $0.14 \pm 0.02$ \\
$\quad$ Without wrist rotation & $1.09 \pm 0.13$ & $0.11 \pm 0.06$ \\
$\quad$ With wrist supination & & \\
\hline
\end{tabular}

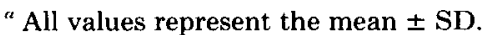

of the movement, with the respective slopes differing little.

The orientation of the handle to be grasped does affect the shape of the trajectories as can be seen by comparing Figure $3, I$ to $L$ with $A$ to $D$. Nevertheless, the slope of the trajectory in velocity space (Fig. $3 K$ ) remains con- stant over a large portion of the deceleratory phase of the movement and its value differs little from the values obtained with the handle oriented vertically. The change in the shape of the trajectory results from the fact that, for a movement to a horizontally oriented handle, the intercept of the trajectory with the $\dot{\theta}$ axis is no longer 0 (Fig. $3 K$ ) as was the case for movements to a handle oriented vertically (Fig. $3, C$ and $G$ ).

The findings illustrated in Figure 3 are typical of the behavior exhibited by all of our subjects. The results are summarized in Table I, which presents the average value of the slope and $\dot{\theta}$ intercept of the trajectories in the deceleratory phase of the movements. The slope was calculated by fitting a straight line over the interval $20 \%$ $<\dot{\theta}<50 \%$ of its maximal value. (There may be abrupt changes in slope for $\dot{\theta}<20 \%$, as in Fig. $3 K$.) The mean values of the slope are all within 1 SD of each other. The intercept is 0 when the handle is oriented vertically. When it is horizontal, instead, the intercept is consist- 
ently positive; the movement is accomplished by initially reaching toward a virtual target located slightly below the handle, and grasping is achieved primarily by forward flexion at the shoulder, resulting in an upward movement at the wrist (see Fig. $3, I$ to $L$ ).

When the $\theta, \phi$ trajectories of Figure $3 J$ are compared with those of Figure $3, B$ and $F$, differences in the overall excursion at the shoulder and elbow joints also can be appreciated. The total change in shoulder angle is somewhat smaller in Figure $3 J$ than in the other two as is the change in elbow angle. Since the final position of the wrist as measured in the $X, Z$ coordinate system is practically the same in all three tasks, the discrepancy in the final value of elbow angle is probably due to the fact that, as was remarked before, reaching movements directed to a vertical target involved some degree of internal rotation and abduction of the upper arm which were compensated by a greater extension at the elbow (or flexion at the shoulder).

Relation between forearm rotation and motion at the proximal joints. The next question that we shall consider is whether fixed relationships exist between the rotation of the forearm and the motions at the elbow and shoulder.
In order to examine this point, we have plotted the former versus either one of the latter. The upper row of Figure 4 shows the relation between shoulder $(\theta)$, elbow $(\phi)$, and wrist $(\psi)$ angles during movements involving pronation, while the lower row shows the same for movements involving supination. Six representative trials are illustrated for each task. As for the $\theta, \phi$ trajectories of the experiment shown in Figure 3, the variability among trials in Figure $4, A$ and $D$ is very small despite the variability in the speed of execution of the movement.

On the contrary, when the changes in wrist angle $(\psi)$ are plotted versus the changes in shoulder angle (Fig. 4, $B$ and $E$ ) or versus the changes in elbow angle (Fig. 4, $C$ and $F$ ), there is considerable intertrial variability. It is obvious already from these few representative trials that no invariant relationship exists between the changes in $\psi$ and the changes in $\theta$ or $\phi$. In most of the individual trajectories plotted, the changes in $\psi$ are restricted to a fraction of the movement at the more proximal joints, but both the timing and duration of wrist movement are extremely variable from trial to trial. Thus, in some trials, the changes in $\psi$ begin almost concomitantly with those in $\theta$ (or $\phi$ ), whereas in other trials, the former lags behind
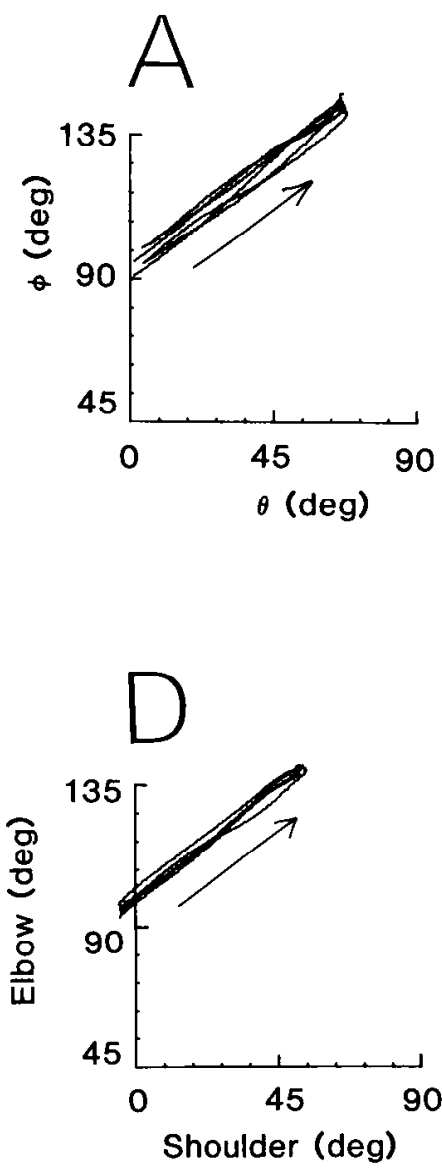
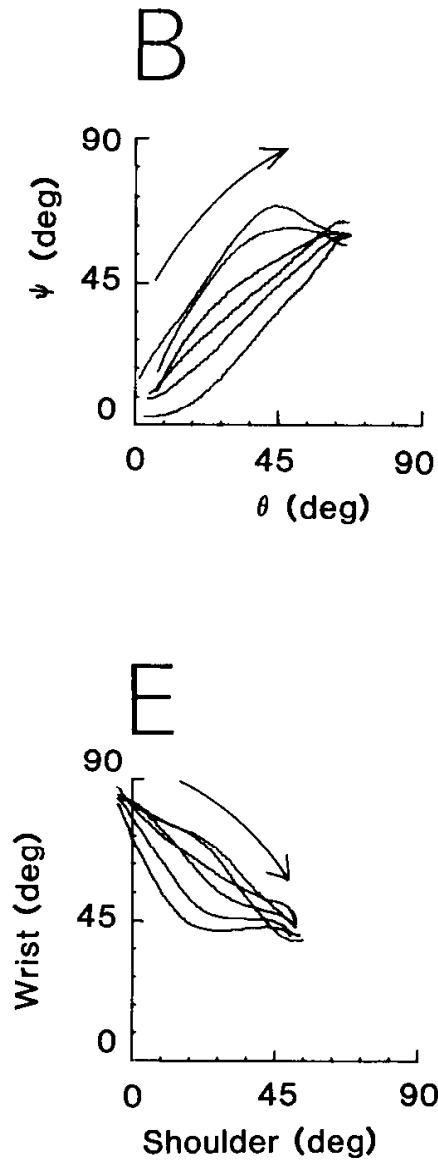
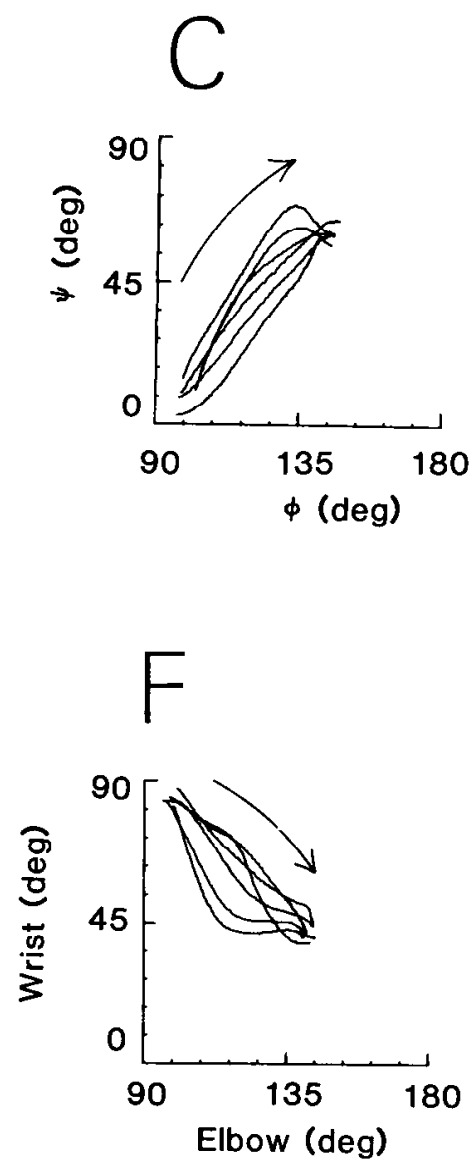

Figure 4. Relations between wrist, shoulder, and elbow motions in a second subject. The upper row ( $A, B$, and $C$ ) shows six representative trials involving pronation, and the lower row ( $D, E$, and $F$ ) shows six trials involving supination. Instantaneous relations between shoulder and elbow angles $(A$ and $D)$, shoulder and wrist ( $B$ and $E$ ), and elbow and wrist ( $C$ and $F$ ) have been plotted for each trial. Maximum angular velocity at the shoulder of these trials was $330,300,200,130,150$, and $130^{\circ} / \mathrm{sec}$ for the pronatory movements (reading from top to bottom in $B$ at $\theta=45^{\circ}$ ) and $140,240,230,170,170$, and $150^{\circ} /$ sec for the supinatory movements (from top to bottom in $E$ at $\theta=30^{\circ}$ ). The arrow represents the direction of movement. 
the latter. Moreover, in some trials, wrist pronation or supination ends when $\theta$ (or $\phi$ ) is only at 50 to $60 \%$ of its total excursion. In other trials, instead, wrist motion occurs mostly during the second half of the movement. Finally, in agreement with the data shown in Figure 2, wrist rotation is, in many instances, biphasic. In one subject, the experiments were repeated twice and, in another subject, four times; the pooled data did not show any consistent family of repeatable trajectories in the $\theta$, $\psi$ (or $\phi, \psi$ ) coordinate system, and the intertrial variability did not decrease with practice.

The plots of Figure 4 neglect the possible influence of movement speed on the variability of the relation between wrist rotation and motion at the other two joints. Even when trials are grouped according to movement speed, there remains a large degree of variability in these relations. However, some general trends emerge. They can be appreciated in Figure 5, where we have plotted the angular velocity profiles of shoulder, elbow, and wrist motions. Single trials have been plotted in ascending order of shoulder velocity after the normalization of movement duration (see "Materials and Methods").
Despite a 4-fold variation in the amplitude of the velocities (and a corresponding decrease in duration of the motion), the profiles of shoulder and elbow angular velocities undergo only minor changes from trial to trial. For slower movements, they are less smooth and have a broader, less defined peak than do those of faster movements. However, the peaks of most traces in each set are almost perfectly aligned. Also, the timing and duration of the motion at the elbow, normalized with respect to the motion at the shoulder, are consistent from trial to trial. Thus, as one would expect from the invariances observed in the velocity space $\dot{\theta}, \dot{\phi}$, the one motion covaries with the other.

On the contrary, motion at the wrist is more variable in timing and duration. Especially for supinatory movements, the time at which forearm rotation is maximal is inconsistent from trial to trial. Furthermore, in many instances, the velocity of supination does not decay smoothly to 0 (see also Fig. $2 B$ ). Nevertheless, a general trend appears in these data. As speed increases, the duration of wrist pronation becomes proportionately shorter than that of motion at the shoulder or elbow.
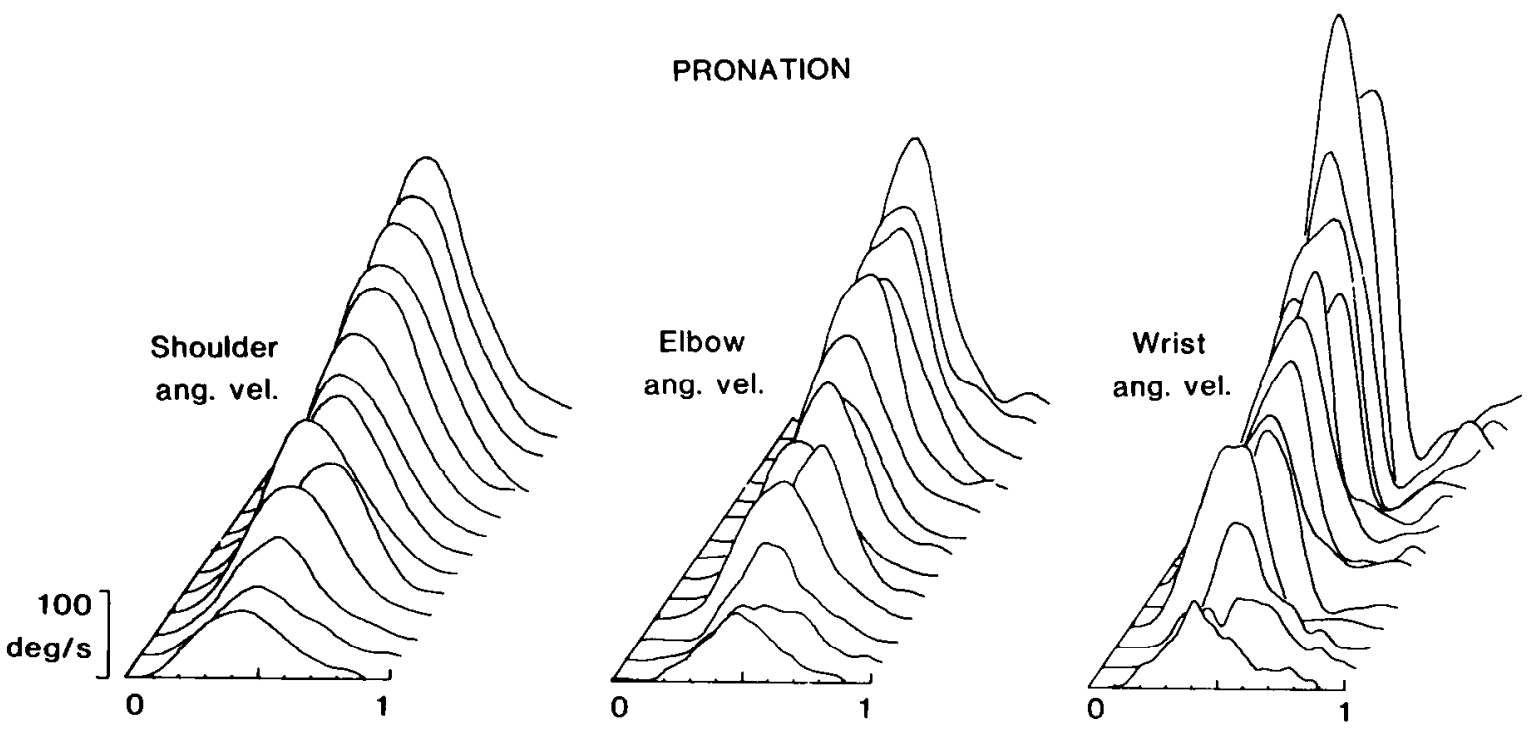

SUPINATION
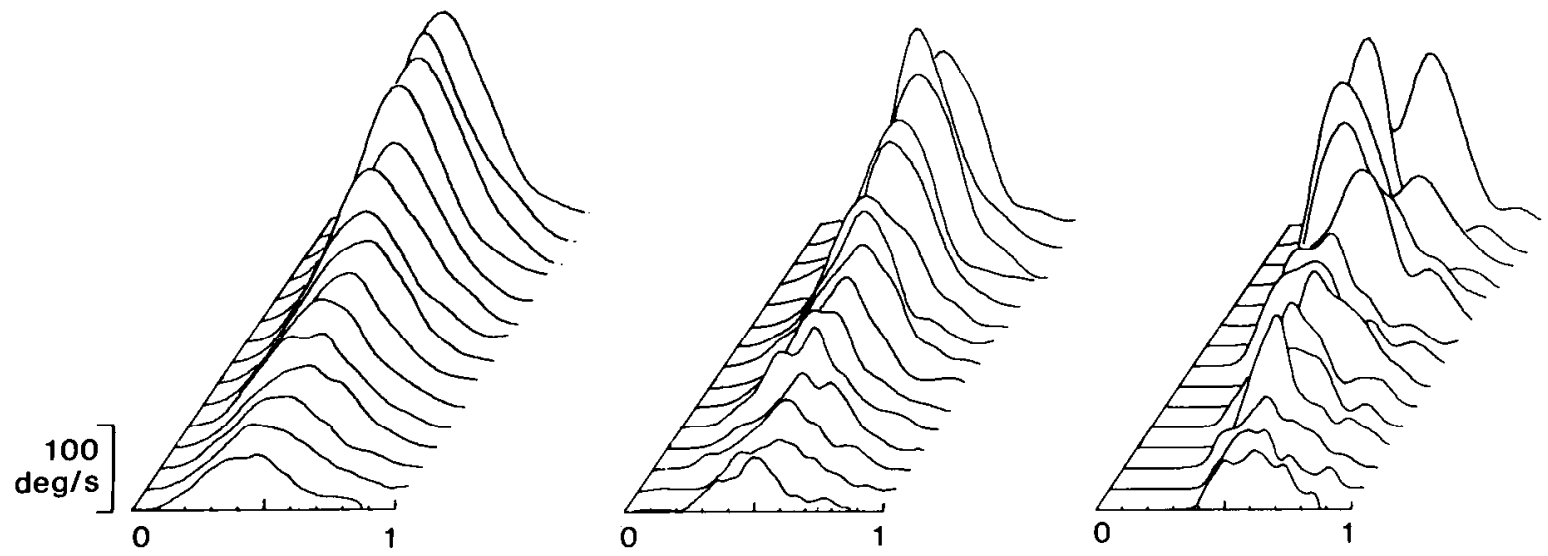

Figure 5. Shoulder, elbow, and forearm angular velocity profiles in a third subject. In the upper row, single trials involving pronation are plotted in ascending order of shoulder velocity; in the lower row, trials involving supination are shown. All trials have been normalized in time relative to the duration of shoulder flexion. Note the shorter duration and the greater variability of wrist motions compared with the other two motions. 
Supinatory movements show the opposite trend. At slower speeds, supination lags behind the onset of the movement at the other joints; as the speed increases, so does the relative duration of wrist supination.

These trends can be appreciated more readily in Figure 6 , where we have schematically illustrated movement duration of shoulder flexion and wrist pronation and supination. All trials from one experiment have been ordered according to maximum shoulder angular velocity. For each trial, the solid circles denote the time of onset and termination of forearm rotation measured relative to the onset of shoulder flexion. The lower and upper bars depict the interval between the onset and termination, respectively, of forearm rotation and shoulder flexion.

Electromyographic activities. Figure 7 shows the average results from the same experiment depicted in Figure 4. The upper row shows movements involving wrist pronation; the lower row depicts supinatory movements. Parts $A$ and $C$ are the averages of the trials performed at an intermediate speed; parts $B$ and $D$ are those of the fastest trials. Figure 8 shows the averages of the fastest trials performed by another subject.

The deltoid exhibits a similar pattern of activity in pronatory and supinatory movements. This pattern is instead clearly dependent upon the speed of movement. In slower movements (Fig. 7, $A$ and $C$ ), the activity in the deltoid increases gradually and then remains at a plateau as required to counteract the force of gravity acting on the arm in its final position. In faster movements (Figs. 7, $B$ and $D$, and $8, A$ and $B$ ), its activity increases more rapidly and there is evidence of an overshoot of the final steady state plateau. The described pattern of activity of the deltoid and its dependence upon speed agree with what has been found previously for simple pointing movements (Soechting and Lacquaniti, 1981).

Unlike deltoid, biceps and pronator teres behave quite differently under the conditions of wrist pronation and supination. Pronator teres discharges abruptly at the onsel of pronatory movements. Furthermore, the increase of its activity is faster than that of deltoid activity, in agreement with the observation that wrist pronation develops faster and ends earlier than shoulder flexion and elbow extension (see also Figs. 5 and 6 ). The pattern of biceps activity depends both on the direction of forearm rotation and on the speed of movement. In the slower supinatory movements (Fig. $7 C$ ), its activity increases slowly to a plateau, while it is more modulated in the faster trials (Figs. $7 D$ and $8 B$ ), with a burst at the onset of the movement followed by a substantial decrease of activity and then by an increase to the final steady state amplitude. This pattern is similar to that previously described for pointing movements (Soechting and Lacquaniti, 1981). The initial burst serves to retard elbow extension which would occur automatically in consequence of the gravitational and the inertial torques produced at the elbow by the acceleration of the shoulder. Instead, for slower movements, the gravitational torque, which builds up more gradually, is dominant (Soechting and Lacquaniti, 1981). Thus, the observed pattern of biceps EMG activity is adequate to account for motion at the elbow. In addition, it is also adequate to account for forearm rotation and the dependence of its time of onset on the speed of the movement (Fig. 6).

The pattern of biceps activity during pronation differs

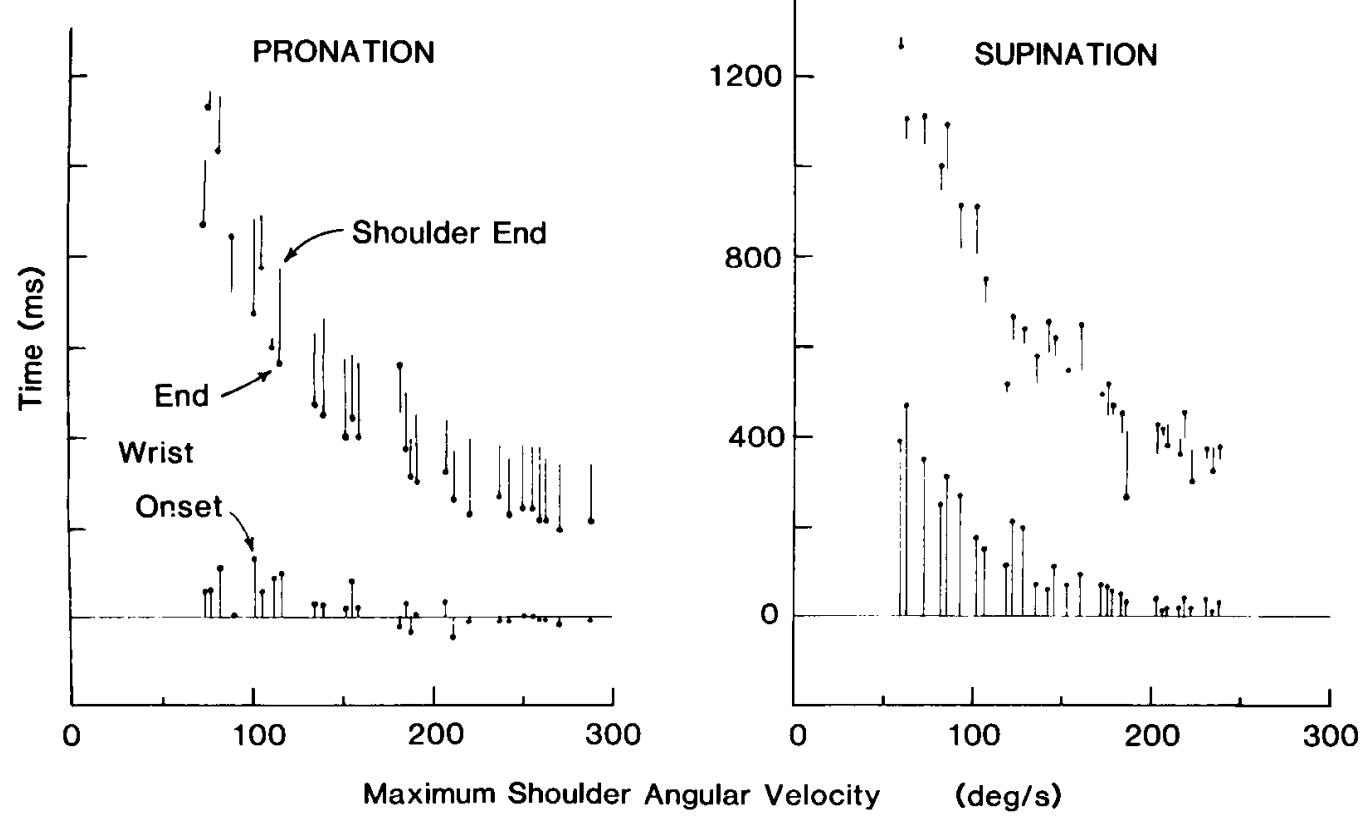

Figure 6. Movement duration and timing of forearm rotation as a function of movement speed at the shoulder. The solid circles denote the time at which forearm rotation begins and ends, with time being measured relative to the onset of shoulder flexion. The upper bars connect the time at which forearm rotation ends to the time at which shoulder flexion terminates. Movement onset and termination are defined as the times at which angular velocity exceeds or drops below $10 \%$ of its maximal value. All trials from one experiment have been ordered according to maximum angular velocity at the shoulder. 

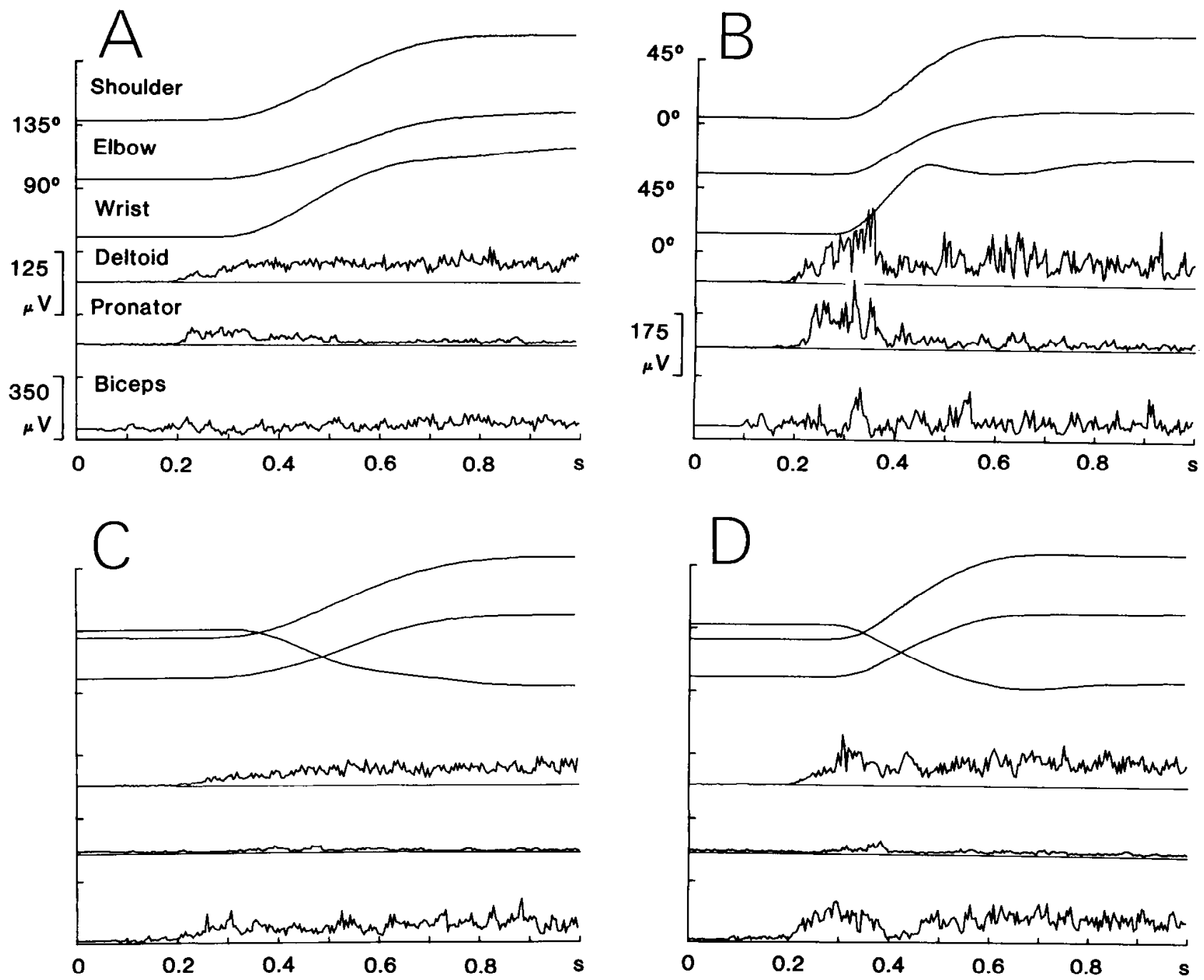

Figure 7. EMG activities during reaching movements. The averages of rectified deltoid, pronator teres, and biceps EMG activities and the average changes in shoulder, elbow, and wrist angles are shown for movements involving pronation $(A$ and $B)$ and supination $(C$ and $D$ ). $A$ and $C$ are the averages of 8 and 6 trials, respectively, obtained at intermediate speed; $B$ and $D$ are those of 3 and 6 trials executed at the fastest speeds. In each panel, the trials were aligned relative to movement onset (defined as the time when shoulder angular velocity exceeds $10 \%$ of its peak value). The calibration for pronator teres amplitude is in $B$. Note the dependence upon speed of EMG patterns of activity and, in biceps and pronator teres, the dependence on the direction of wrist movement.
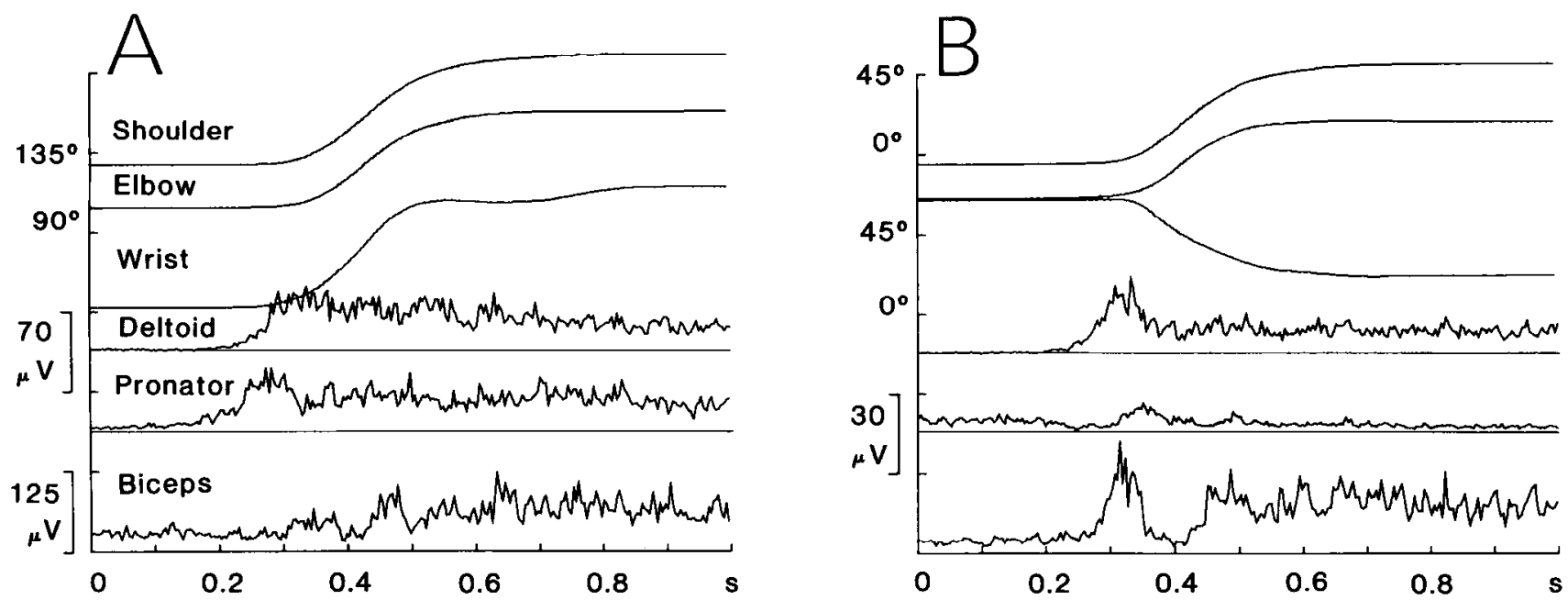

Figure 8. EMG activities in another subject. These averages are of the fastest movements produced by this subject. $A$ is the average of 11 trials involving pronation; $B$ is that of 11 trials involving supination. 
considerably from that observed during supination or in the absence of forearm rotation. The initial burst of activity is no longer present during faster movements and, on the whole, the amount of modulation is much less than during supinatory movements. Since motion at the elbow is the same during supinatory and pronatory movements, the observed biceps activity during pronation cannot account for the torque required to produce that motion. Therefore, one can deduce that, in this case, activity in other elbow flexors substitutes for that of biceps to produce the same motion.

\section{Discussion}

We have presented an analysis of arm movements which involved reaching for and grasping a stationary object and required shoulder flexion, elbow extension, and wrist pronation or supination. In agreement with previous results obtained with pointing movements, we found that the angular motion at the elbow is related consistently to the motion at the shoulder, with the relationship remaining invariant over a wide range of speeds of movement. This finding also agrees with the observation of Georgopoulos et al. (1981) that the spatial variability of pointing movements performed by trained monkeys was small. Further, the concomitant presence of motion at the wrist does not affect the particular shape of the $\phi, \theta$ trajectory of movements directed to a given target.

On the contrary, no such invariant relationship exists between forearm rotations and the motions at the two more proximal joints. This difference cannot be ascribed to the external constraints imposed by the tasks, since these constraints were the same for the motions both at the proximal joints and at the wrist and consisted simply in the specification of the initial and final angular positions. We believe instead that our findings imply that shoulder and elbow motions are coupled functionally, whereas wrist motion is uncoupled from the other two, and that this difference may result from the nature of the internal constraints acting on the motions at the two proximal joints and on wrist motion.

The first of such constraints is that the angular motion at the elbow is coupled inertially with the angular motion at the shoulder but not with that at the wrist. By inertial coupling, we mean that a torque applied solely at one joint, such as that produced by muscle contraction, will result in angular motion both at the shoulder and at the elbow. In this sense, forearm rotation is uncoupled inertially from shoulder or elbow flexion-extension because their respective planes of movement are perpendicular to each other. Thus, in the presence of inertial coupling, the advantage of having a functional relation between shoulder and elbow motions may be that the complexity of the control problem is reduced. The torque at the shoulder (or elbow) required to produce the movement, which would be otherwise a function of both shoulder and elbow angles and their derivatives, becomes a function of a single variable.

One of the invariant relations which characterize shoulder and elbow motions is that the slope of the trajectory in $\dot{\theta}, \dot{\phi}$ is constant over a considerable portion of the deceleratory phase of the movement and has the same value in all of the tasks. This value (close to unity) is the same as that found in the previous experiments involving simpler pointing movements with only two degrees of freedom (Soechting and Lacquaniti, 1981). The fact that the slope of $\dot{\theta}, \dot{\phi}$ is constant and that the orientation of the target only effects a shift in the $\dot{\theta}, \dot{\phi}$ trajectory means that the ratio between the angular acceleration is the parameter which is invariant of target location. Therefore, one can hypothesize that $\ddot{\theta}$ and $\ddot{\phi}$ are the jointly regulated variables in the terminal phase, the advantage being that of a considerable economy of function (Gelfand et al., 1971; Greene, 1972). We have suggested previously (Soechting and Lacquaniti, 1981) that this invariant relation could result from a feedback regulation of the movement involving force feedback from peripheral receptors. In particular, the fact that the same motion at a joint is achieved automatically with a different parcellation among the activities of agonists involved in producing the movement (Figs. 7 and 8 ) would point to the need for proprioceptive feedback as the most economical way of regulating the kinematic variables.

Notice that, as a consequence of the coupling between shoulder and elbow motions, the number of degrees of freedom of the translational movement of the hand along the parasagittal plane involved in the reaching task also is reduced from two to one. Therefore, the spatial trajectory of the hand, which would be $a$ priori indeterminate since only the initial and final positions were experimentally constrained, becomes determinate and is, in fact, highly reproducible from trial to trial of each task irrespective of the speed of execution.

The described kinematic behavior at the shoulder and elbow has been verified in the context of unidirectional movements occurring in the vertical plane and involving shoulder flexion and elbow extension. Morasso (1981) recently has described movements occurring in a horizontal plane (therefore under the constant influence of gravity). When the movement required shoulder flexion and elbow extension, his results were comparable to ours. However, movements involving extension at both joints or shoulder extension and elbow flexion exhibited angular velocity profiles which were not single peaked nor did the maximum velocity at shoulder and elbow coincide.

Unlike shoulder and elbow motions, forearm rotations proved to be extremely variable in timing, course, and duration. One could argue that wrist motion is controlled separately from the other two motions because, at least in this particular task, there is no need for, nor special advantage in, having forearm rotation develop synchronously with the motions at the other two joints. In fact, we observed that the duration of wrist motions, although variable, was generally shorter than that of the motions at shoulder or elbow. This could be only because the moment of inertia for forearm rotation is much less than that for elbow or shoulder motions, and therefore, the same torque applied at the wrist or at the elbow will produce a larger angular acceleration at the former than at the latter. Thus, forearm rotations theoretically could take place indifferently at variable times during the reaching task and still remain within the time limits imposed by the slower motions at the elbow and shoulder.

However, forearm rotation, although not invariantly 
related to the motion at the elbow, is not independent of it. There exists, in fact, another internal constraint, namely the biomechanical linkage between wrist and elbow motion established by the presence of bifunctional muscles acting on both joints. Most of the muscles which are involved in pronation or supination act also as either flexors or extensors of the elbow (Basmajian, 1978). Our data show that the pattern and amplitude of the activity of bifunctional muscles reflect not only the movement required at the elbow but also that required at the wrist. This observation lends further support to the hypothesis (Soechting and Lacquaniti, 1981) that it is the movement itself, and not the pattern of activity in individual muscles, which is invariant during compound motion of the arm. Indeed, if the relationship between the kinematic variables of shoulder and elbow is regulated in the reaching task, then the activities of the muscles involved will be set so as to maintain that kinematic invariance. Note that this does not imply a unique pattern of activity for a given muscle. Instead, this statement only implies that activity is distributed among the synergists and antagonists in such a manner that the net torque produced is appropriate. As we have shown, the pattern of activity in a given muscle (e.g., biceps) may change without affecting the observed invariant kinematics (Figs. 7 and 8). (A dependence of muscle activities on the direction of fast horizontal reaching movements also has been described by Wadman et al., 1980.) Consequently, provided that wrist pronation or supination is carried out within some temporal limits, the pattern of activity of bifunctional muscles (acting in wrist and elbow motions) will be regulated so as to satisfy the constraints of shoulderelbow functional coupling, and, as a result, wrist motion will be functionally subordinate to that of the other two joints.

As a last point, the invariance of shoulder and elbow kinematics under changes in the speed of the reaching movement and despite changes in the pattern, amplitude and, probably, even participation of the pertinent muscles can be related to the concept of motor equivalence (Lashley, 1930; Hebb, 1949; Bernstein, 1967; Gelfand et al., 1971; Greene, 1972; Hughes and Abbs, 1976; Terzuolo and Viviani, 1979; Viviani and Terzuolo, 1981). One interpretation of the phenomenon of motor equivalence is that the construction of a motor act involves different hierarchical levels of organization. At the higher levels, the general structure of a motor act would be defined in abstract terms whose features are only those properties of the motor act which remain invariant under different kinds of spatiotemporal transformations. At the lower levels of organization, more contingent parameters of the movement (such as the muscles required and their pattern of activation) would be defined on the basis of specific task demands (for instance, the speed of execution) and of the current state of the environment and musculoskeletal apparatus. Our data seem to suggest that these two stages in the construction of a motor act cannot be conceived as entirely independent processes. The existence of peripheral constraints in the motor apparatus (such as the bifunctional nature of most of the pronators and supinators or the inertial coupling between shoulder and elbow motions) will influence the determination of the invariant properties of the motor act. How- ever, when the specific goal of a motor task requires a consistent temporal coordination between motions which would otherwise be variably related (for example, handwriting), such coordination may be enforced upon the effector even perhaps at the expense of other invariant relations of the movement.

\section{References}

Basmajian, J. V. (1978) Muscles Alive, Ed. 4, p. 495, Williams \& Wilkins, Baltimore.

Bernstein, N. (1967) The Co-ordination and Regulation of Movements, p. 196, Pergamon Press, Oxford.

Bishop, A., and A. Harrison (1977) Kots and Syrovegin (1966)A demonstration of modular units in motor programming? $\mathrm{J}$. Hum. Move. Stud. 3: 99-109.

Chao, E. Y. (1978) Experimental methods for biomechanical measurement of joint kinematics. In CRC Handbook of Bioengineering in Medicine and Biology, Vol. 1, pp. 385411, CRC Press, Inc., West Palm Beach, FL.

Chao, E. Y. (1980) Justification of triaxial goniometer for the measurement of joint rotation. J. Biomech. 13: 989-1006.

Chao, E. Y., K. N. An, L. J. Askew, and B. F. Morrey (1980) Electrogoniometer for the measurement of human elbow joint rotation. J. Biomech. Engr, 102: 301-310.

Dvir, Z., and N. Berme (1978) The shoulder complex in elevation of the arm: A mechanism approach. J. Biomech. 11: 219225.

Gelfand, I. M., V. S. Gurfinkel, M. L. Tsetlin, and M. L. Shik (1971) Some problems in the analysis of movements. In Models of the Structural-Functional Organization of Certain Biological Systems, I. M. Gelfand, V. S. Gurfinkel, S. V. Fomin, and M. L. Tsetlin, eds., pp. 329-345, MIT Press, Cambridge, MA.

Georgopoulos, A. P., J. F. Kalaska, and J. T. Massey (1981) Spatial trajectories and reaction times of aimed movements: Effects of practice, uncertainty and change in target location. J. Neurophysiol. 46: 725-743.

Greene, P. H. (1972) Problems of organization of motor systems. Prog. Theor. Biol. 2: 304-338.

Hebb, D. O. (1949) The Organization of Behavior. A Neuropsychological Theory, Wiley, New York.

Hughes, O. M., and J. H. Abbs (1976) Labial-mandibular coordination in the production of speech: Implications for the operation of motor equivalence. Phonetica 33: 199-221.

Kots, Y. M., and A. M. Syrovegin (1966) Fixed sets of variants of interactions of the muscles of two joints used in the execution of simple voluntary movements. Biofizika 11: 10611066.

Lashley, K. S. (1930) Basic neural mechanisms in behavior. Psychol. Rev. 37: 1-24.

Morasso, P. (1981) Spatial control of arm movements. Exp. Brain Res. 42: 223-237.

Soechting, J. F., and F. Lacquaniti (1981) Invariant characteristics of a pointing movement in man. J. Neurosci. 1: 710720.

Terzuolo, C., and P. Viviani (1979) The central representation of learned motor patterns. In Posture and Movement, R. E. Talbott and D. R. Humphrey, eds., pp. 113-121, Raven Press, New York.

Viviani, P., and C. Terzuolo (1981) The organization of movement in handwriting and typing. In Language Production. Vol. II: The Production of Language in Non-Speech Modalities, B. Butterworth, ed., Academic Press, New York, in press.

Wadman, W. J., J. J. Denier van der Gon, and R. J. A. Derksen (1980) Muscle activation patterns for fast goal-directed arm movements. J. Hum. Move. Stud. 6: 19-37. 